\title{
El Panamericanismo y otras formas de relaciones internacionales en las Américas en las primeras décadas del Siglo XX
}

Norberto O. Ferreras ${ }^{1}$

\section{Resumo:}

Neste artigo analisamos as relações internacionais americanas considerando as formas de relacionamento entre os diversos países. Ao longo do artigo apresentamos as distintas possibilidades de pensar e organizar essas relações estabelecendo seus eixos prioritários: os Estados Unidos (Pan-americanismo); a Espanha (Hispanismo) e os próprios países latino-americanos (Latino americanismo). Também consideramos a relação estabelecida entre os países que faziam parte da União Pan-americana, entendendo esta como um espaço de diálogo, antes que de confronto. E finalmente como o Pan-americanismo se integrou no sistema genebrino da Sociedade de Nações.

Palavras-Chave: Relações Internacionais; Sociedade de Nações; Pan-americanismo

\begin{abstract}
:
In this article we analyze the American international relations considering the forms of relationships between the various countries. Throughout the article we present the different possibilities of thinking and organizing these relationships setting their priorities: the United States (Pan-Americanism), Spain (Hispanicism) and own Latin American countries (Latin Americanism). We also consider the relationship between the countries that were part of the Pan American Union understood this as a space for dialogue instead of confrontation. And finally as the Pan-Americanism has integrated into the League of Nations system.
\end{abstract}

Key words: International Relations; League of Nations; Pan-Americanism

Artigo recebido em: 25/06/2013

Artigo aprobado em: 09/09/2013

\footnotetext{
${ }^{1}$ Professor do Departamento de História da Universidade Federal Fluminense (UFF), Brasil. Pesquisador 2 do CNPQ. E-mail: ferreras@ vm.uff.br

Revista Eletrônica da ANPHLAC, ISSN 1679-1061, n.15, p. 155-174, jul./dez. 2013. 


\section{1.- Introducción}

Desde inicio de su vida independiente los países americanos han intentado llegar a formas de convivencia que no estuvieran basadas en los conflictos militares. En rarísimas ocasiones las soluciones no fueron por la vía de la negociación y de los acuerdos entre las partes, no siempre satisfactorios. Los pocos conflictos internacionales, la mayoría de los cuales tuvieron lugar en el siglo XIX, tuvieron consecuencias dramáticas para los involucrados y para los vecinos. Basta recordar la Guerra del Paraguay (1864-1870); la Guerra del Pacífico (1879-1883); la Guerra del Chaco (1932-1935) y la Guerra del Fútbol (1970) como para pensar en la dimensión que las guerras alcanzaron y sus efectos, generalmente nocivo para todas las partes involucradas. Al mismo tiempo podemos comparar con otras regiones del planeta y vemos que los conflictos intrarregionales son pocos. De alguna forma estos conflictos conformaron la prioridad de una matriz de negociación al interior de la región. En este dialogo tenemos que incorporar a los Estados Unidos, a pesar de sus múltiples intervenciones en varias partes de América, a veces por mano propia, en otras por sus emisarios.

La incorporación de los Estados Unidos en este artículo se debe a que de alguna forma los esfuerzos para construir un ámbito de debate también estuvieron direccionados a que los Estados Unidos fuese un interlocutor confiable, pero también la oportunidad de este país de que su importancia fuese reconocida por el resto del continente.

Cuando estudiamos las relaciones internacionales interamericanas se suceden y coexisten algunos paradigmas comportamentales que varían según las circunstancias y las posiciones relativas que ocupa cada una de las naciones. En este sentido los países del hemisferio americano pasaron por diversas fases, desde el aislamiento individual a la integración plena global, que en la mayoría de los casos no representa una posición necesariamente ideológica definitiva y si una forma de posicionarse, voluntariamente $u$ obligadamente, en diferentes momentos de su Historia, en relación a los otros países del continente, siguiendo sus propios intereses o según las posibilidades y capacidad para defender esos intereses. Entendemos que los intereses nacionales son definidos colectivamente y en un determinado momento y que no son inmutables, por el contrario estos están históricamente datados.

Es por eso que para poder comprender las posiciones que los países americanos tomaron, así como sus idas y vueltas en relación a las mismas, tenemos que analizar las

Revista Eletrônica da ANPHLAC, ISSN 1679-1061, n.15, p. 155-174, jul./dez. 2013. 
relaciones entre los países de América Latina, las relaciones con los Estados Unidos y con los países europeos que lideraban ambas organizaciones, Inglaterra y Francia. Los vínculos de estos países son necesariamente relacionales, o sea que dependen más de la dinámica y de los intereses del momento, y por lo tanto dependen de una serie de factores que condicionan sus posicionamientos. También tenemos que considerar el hecho de que muchas veces los factores correspondientes a la política interna influyeron decisivamente en las posiciones de los distintos gobiernos. La transitoriedad de las posiciones depende, también, de la duración y necesidades de los gobiernos. Debemos pensar que cada gobierno es un campo de fuerzas en el que existen tensiones que llegan desde el exterior. De esta forma, no podemos pensar en posiciones internacionales que sean inmutables y si temporarias y de acuerdo a las correlaciones de fuerzas de las alianzas gobernantes.

En este artículo analizaremos con mayor detenimiento un periodo de las relaciones internacionales e intentaremos presentar como las mismas se configuraron a lo largo de la década de 1930 según intereses propios y coyunturales. La inestabilidad del periodo nos permite visibilizar algunas líneas generales del relacionamiento y pensar en políticas que anteceden a los protagonistas, pero, que con mucha dificultad los sucederán. La situación de las décadas siguientes marcadas por las disputas ideológicas entre capitalismo y comunismo, serán diferentes en cuanto a los objetivos propuestos y a las posibilidades de autonomía. La década de 1930 abrió una serie de posibilidades que no necesariamente se concretaron, como las alternativas abiertas por el New Deal, las teorías corporativas, el pensamiento social de la Iglesia y las experiencias socialdemócratas europeas, principalmente la francesa.

Si tomamos como ejemplo a la Argentina, vemos que el neutralismo que este país pasó a practicar desde la Guerra del Paraguay se debía a la imposibilidad de poder conformar al conjunto de los sectores dominantes y también de los grupos subalternos que se dividían y disputaban la influencia de los países europeos y limítrofes. Por lo tanto, la posición neutralista y antibélica generó consensos que permitieron la coexistencia de grupos de adversarios al interior del Estado, preservando los negocios de exportación a nivel internacional. Durante buena parte del Siglo XX el neutralismo permitió que los grupos dominantes de Argentina no tuvieran que explicitar su vínculo con Inglaterra, Francia o Alemania, en cuanto que los Estados Unidos no era una alternativa comercial para los productos argentinos. Así el neutralismo era tanto una política que preservaba las exportaciones, como era una forma de mantener una Revista Eletrônica da ANPHLAC, ISSN 1679-1061, n.15, p. 155-174, jul./dez. 2013. 
posición crítica en relación a las políticas expansionistas estadunidenses en el resto de América. Cuando la neutralidad pasó a favorecer claramente a alguno de los sectores esta política entró en entredicho.

Es común la afirmación de que el Golpe de 1943 en la Argentina fue coordinado por el Grupo de Oficiales Unidos (GOU), entre los que se encontraba Juan Domingo Perón, y que este grupo era pro nazi y que por eso su posición era la de la neutralidad durante la Segunda Guerra Mundial para permitir que Alemania tuviera continuidad en el abastecimiento de alimentos. Esta lectura tiene como objetivo asociar el peronismo al nazismo o al fascismo dejando de lado el hecho de que la neutralidad antecedió al GOU en el gobierno y que la misma favorecía a los grupos de exportadores de carnes y cereales que tenían en el Reino Unido a su principal mercado. La continuidad de la neutralidad después de la liberación de los mares y con el avance de la guerra prácticamente no alteraba la relación de fuerzas entre las potencias contendientes, pero permitía identificar a aquellos que no se posicionaban contra el Eje y a favor de los Aliados (MACDONALD, C. A. 1980, pp. 383 e 384). ${ }^{2}$ Una segunda lectura recoloca la cuestión económica y muestra que el neutralismo inhibía a los intereses agrarios de beneficiarse de la coyuntura y favorecía a los industriales que se favorecían con el aislamiento (MACDONALD, C. A. 1980, pp. 385 e 396).

El primer neutralismo, a pesar de cuestionado por los Estados Unidos, no fue necesariamente estigmatizado. El segundo neutralismo, a partir de 1943 cuando el GOU llega al poder por la vía de un Golpe de Estado, fue visto como una amenaza y una falta de compromiso con las democracias occidentales, a pesar de que muchos de los apoyos a las democracias europeas no fuesen de países necesariamente democráticos, como era el caso de la Nicaragua de Somoza.

Por eso entendemos que las posiciones están vinculadas a los momentos políticos o económicos de los países y que no pueden ser vistos como posiciones inalterables a lo largo del tiempo. Para decir que los Estados Unidos mantienen una posición de aislamiento de la política internacional, se pone como ejemplo que este país no adhirió a la Sociedad de Naciones. Lo que debemos resaltar en este caso es que la no adhesión a la SdN está directamente relacionada con cuestiones de política interna y no con una falta de interés internacional (MACMILLAN, 2002, pp. 488 a 493). ¿Cómo

\footnotetext{
${ }^{2}$.- En este mismo artículo MacDonald muestra que la relación del Eje con la Argentina no estaba relacionada con el gobierno en el que participaron facciones del GOU como aparece en las páginas 388 a 390.
}

Revista Eletrônica da ANPHLAC, ISSN 1679-1061, n.15, p. 155-174, jul./dez. 2013. 
podríamos afirmar que los Estados Unidos "vuelven a su tradicional política de aislamiento" cuando ese país acababa de salir de una Guerra definida como "Mundial"? ¿Cómo se explica el aislamiento cuando la SdN fue una propuesta del propio presidente de los Estados Unidos, Woodrow Wilson? En todo caso podríamos afirmar que los Estados Unidos abandonaron las cuestiones políticas europeas frustrados por la falta de compromiso de los demás países vencedores en construir alternativas de convivencia. Tampoco podemos mantener la afirmación relacionada al aislamiento cuando este país se involucra en una serie de intervenciones militares en América Latina. En este caso la mención al neutralismo implica reconocer un status de relación de necesidad entre las políticas de los Estados Unidos y América Latina, más específicamente con el Caribe y un menor interés por la política europea que en el inicio de la gestión Wilson.

Finalmente, precisamos recordar que no estamos aquí estableciendo relaciones fijas y que tenemos que tener en cuenta los contextos en que se producen estos vínculos, considerando las relaciones de colaboración y/o competición que se dan entre los diversos países. También no podemos dejar de considerar que las instituciones internacionales surgidas a raíz del Tratado de Paz de Versalles también transformaron sus objetivos y prácticas a partir de sus propias necesidades. Tanto la Sociedad de Naciones como la OIT variaron sus posiciones en relación a los países latinoamericanos una vez que el contexto internacional se modificó con el ascenso de los fascismos y la consiguiente amenaza para las democracias atlánticas.

Estas son algunas de las preocupaciones centrales de este artículo e intentaremos compatibilizar estas cuestiones con la relación existente entre el panamericanismo, el latino-americanismo y el universalismo propuesto por la OIT.

\section{2.- Panamericanismo, Latino-americanismo, Hispanoamericanismo}

Para analizar esta política internacional que hemos denominado de "relacional" tenemos que analizar algunos elementos básicos en los vínculos entre los distintos países que conforman la región. En principio hay una fuerte tendencia a considerar el panamericanismo como una forma de consolidar la hegemonía de los Estados Unidos sobre el resto del continente (BERGER, 1993, p. 1). Sin embargo, el panamericanismo se mostró como un espacio fértil para la presentación de diferencias y reclamos. En ese sentido las conferencias panamericanas también pueden ser vistas como el lugar del diálogo o de la sobreactuación de las diferencias antes que como un espacio de conflicto. Que los Estados Unidos intentasen controlar las reuniones o que fuesen el

Revista Eletrônica da ANPHLAC, ISSN 1679-1061, n.15, p. 155-174, jul./dez. 2013. 
espacio de demostración de su poder no implica que este fuese el resultado final de las mismas o que los demás países simplemente lo aceptasen. En este espacio el resto de los países de la región podía negociar las condiciones existentes cuanto oponerse rotundamente a las iniciativas estadunidenses. ${ }^{3}$

El panamericanismo como forma de organización de las relaciones entre los países americanos data del final del Siglo XIX. Sin embargo no podemos olvidarnos que las tentativas de reunión y de organización de los países americanos preceden a estos encuentros. Es cierto que existieron dificultades para estas reuniones, entre ellas las desconfianza y/o la falta de logística adecuada, lo que acababa dificultando la participación de varios países de estos encuentros pero no por eso debemos dejar de mencionarlos. Las tentativas de reunir a los países americanos se remontan al Congreso Anfictiónico organizado por Bolívar en 1826, el más ambicioso y con mayor participación del período anterior a 1890. Este congreso contó con la presencia de representantes de los Estados Unidos, México, Confederación Centroamericana, Perú, Bolivia y Gran Colombia. ${ }^{4}$ Posteriormente se reunieron el Primer Congreso Internacional Americano en Lima (1847); el Congreso Continental en Santiago de Chile (1856); y el Segundo Congreso Internacional Americano, nuevamente en Lima (1865). Estos encuentros eran ambiciosos en sus objetivos y pobres en los resultados pero evidenciaban un interés regional por establecer vínculos duraderos.

El panamericanismo, como forma de asociación y organización, debe ser considerado como una larga tradición en las relaciones internacionales entre los países del continente americano. La Primera Conferencia Interamericana, la más extensa de todas, tuvo lugar en Washington y se inició en octubre de 1889, finalizando en abril de 1890. La Décima y última conferencia fue realizada en Caracas en 1954 y sirvió de plataforma para la creación de la actual Organización de los Estados Americanos (OEA). Para esta presentación es importante resaltar que durante la década de 1930 se realizaron tres conferencias: la VII en 1933 en Montevideo; la VIII en Lima en 1938; y un encuentro especial denominado de Conferencia Interamericana de Consolidación de la Paz que tuvo lugar en Buenos Aires, en 1936, y que contó con la presencia del presidente de los Estados Unidos, Franklin Delano Roosevelt.

\footnotetext{
3.- Para algunas de las idas y vueltas de este panamericanismo critico o conciliador ver GRANDIN, 2006, pp. 1043 e 1051.

4.- La Gran Colombia estaba conformada por los actuales territorios de Colombia, Panamá, Venezuela y Ecuador. La Confederación Centroamericana, por su vez, integraba los actuales territorios de Guatemala, El Salvador, Honduras, Nicaragua y Costa Rica. Para los detalles de estos encuentros ver MORGENFELD, 2011, pp. 39 a 60.

Revista Eletrônica da ANPHLAC, ISSN 1679-1061, n.15, p. 155-174, jul./dez. 2013.
} 


\section{CONFERENCIAS PAN-AMERICANAS}

1) Washington, D.C., 1889-1890

2) México DF, 1901-1902

3) Rio de Janeiro, 1906

4) Buenos Aires, 1910

5) Santiago de Chile, 1923

6) La Habana, 1928

7) Montevideo, 1933

8) Lima, 1938

9) Bogotá, 1948

10) Caracas, 1954

Para completar el cuadro de reuniones que tuvieron el carácter de encuentros de Estado tenemos que agregar la ya mencionada Conferencia Interamericana de Consolidación de la Paz de 1936 en Buenos Aires y la Conferencia Interamericana para la Manutención da Paz y la Seguridad del Continente de 1947 en Rio de Janeiro. Hay también una miríada de otros encuentros técnicos o sectoriales, como por ej. las temáticas vinculadas con la salud, las comunicaciones o el servicio postal, que dieron mayor densidad a este panamericanismo de diálogo y acuerdos.

Pero este panamericanismo no fue la única forma en que se relacionaron los dos hemisferios de las Américas. Desde la formación de los Estados Unidos de América y a lo largo del Siglo XIX y XX no deparamos con la adaptación de la Doctrina Monroe como una teoría de la superioridad del Norte, el recurso a amenazar con el uso de la fuerza militar para resolver conflictos o defender los intereses de los Estados Unidos y también con varias intervenciones armadas formales o informales. Por lo tanto, las reuniones panamericanas permitían colocar a los Estados Unidos en una posición de negociación antes que a la defensiva o en la ofensiva militar. ${ }^{5}$

Como mencionamos anteriormente, el panamericanismo no es una teoría fija, debe ser visto más como una práctica política. ${ }^{6}$ Lo que define al panamericanismo es el

\footnotetext{
5.- Para un análisis de las posiciones de los Estados Unidos en relaciona a América Latina a lo largo del Siglo XIX e inicios del Siglo XX en relación a las acciones pacificas o militares ver los capítulos 5 (Control) y 6 (Intervention) de SEXTON, 2011.

${ }^{6}$.- Es posible identificar algunos momentos en la relación entre los Estados Unidos y América Latina. Carlos Oliva Campos entiende que hay cinco fases de hegemonía estadunidense y el periodo que Revista Eletrônica da ANPHLAC, ISSN 1679-1061, n.15, p. 155-174, jul./dez. 2013.
} 
hecho de priorizar las relaciones entre los países del continente, como una forma de establecer y delimitar los vínculos que deberían primar entre los países que forman parte del continente. En el panamericanismo el vínculo principal sería con los Estados Unidos y este país mediaría la relación con el resto. Pero el dominio continental no aparecía como el motor principal del mismo, por el contrario, el panamericanismo estaba basado en los elementos comunes de la región, como por ej.: el hecho de tener una Historia y una geografía en común, así como el de tener las mismas ideas sobre República, Libertad y Democracia. Esta era la forma en que los Estados Unidos presentaban su forma de relacionarse con los demás países del continente. Esto se complementaba con los reclamos de pacificación y estabilidad que hacia este país y que eran mucho más importantes para sus propios intereses económicos y comerciales, porque le permitía expandir sus actividades económicas de forma confiable. El panamericanismo, para los Estados Unidos, era visto tanto como una forma de expandir su influencia como detener cualquier avance europeo en la región, fuese militar o fuese comercial, que les disputase el dominio regional. ${ }^{7}$

El panamericanismo tenía sus contrapartidas para varios de los países del resto del continente: garantizaba compradores para sus productos y un abastecimiento constante, así como un apoyo internacional en disputas con países exteriores al continente. Siempre de parte de los Estados Unidos.

Pero el panamericanismo no era, ni es, la única forma de comprender y de pensar la región. Por un lado tenemos el latino-americanismo, como una manera de relacionarse entre los países de América Central y del Sur, fuera de la influencia decisiva de los Estados Unidos. El latino-americanismo fue un producto de la intelectualidad francesa que rescataba la tradición católica como opuesta al protestantismo del Norte. Y con el catolicismo se priorizaba la virtud sobre la utilidad, la belleza sobre la utilidad y la raza latina sobre la anglosajona. El modelo cultural, y con él el económico, se centraba en Francia, el farol cultural del Siglo XIX. Esta era una forma de abrir las puertas al capital y al comercio de este origen. La versión francesa del latino-americanismo colocaba a la cultura como una forma de diplomacia y de inserción regional (BETHELL, 2010, p. 457).

estudiamos lo denomina de Etapa Ajuste Hegemónico (1898 a 1945) y al interior de este periodo podemos verificar las políticas de Big stick, la diplomacia del Dólar y la política de Buena Vecindad, por lo tanto, es difícil entender esta hegemonía como monolítica, sino como producto de la lectura realizada por los diplomáticos americanos en cada momento especifico. OLIVA CAMPOS, 2000, p. 241.

7.- Para más detalles sobre estas cuestiones ver BETHELL, 2010, p. 464.

Revista Eletrônica da ANPHLAC, ISSN 1679-1061, n.15, p. 155-174, jul./dez. 2013. 
El latino-americanismo permitía que la América Portuguesa de Brasil y la francesa de Haití y las demás colonias del Caribe pudiesen ser incorporadas a un destino en común con el resto de la región sin la influencia de los Estados Unidos. Cierto es que esta corriente se expresó en las Conferencias Panamericanas pero nunca buscó una organización propia.

Claro está que este latino-americanismo tiene un pecado original y es el hecho de que fue utilizado como justificativa para la invasión francesa y la entronización del austriaco Maximiliano de Habsburgo como Emperador de México en 1864. En teoría la Francia imperial de Napoleón III socorría a sus hermanos mexicanos contra el gigante del Norte en defensa de la religión católica y de la raza latina, proporcionando la estabilidad de una monarquía. La operación resultó en un fracaso doble para Francia tanto de su diplomacia como de su ejército. El latino-americanismo había hecho un debut tan imperial y agresivo como podrían haber sido los Estados Unidos y sus disputas con el mismo México. Por lo que demoraría a ser revisitado.

Si la idea de la latinidad se volvió atractiva para los iberoamericanos se debió más a la falta de opciones y a la continuidad de Francia como una alternativa cultural y económica, aun después de la derrota en la Guerra Franco-Prusiana en 1871. Las tradiciones, cultura, lengua y religión de los latinos no eran necesariamente inferiores a las de los estadunidenses. Las críticas a la cultura y sociedad de la América del Norte desarrolladas por Alexis de Tocqueville en su obra más conocida: "La democracia en América" (TOCQUEVILLE, 1985), permitían rever la supremacía americana e iniciaba una matriz de pensamiento crítica a los del norte que fortalecía la imagen de una latinidad que no precisaba sentirse en inferioridad de condiciones.

La latinidad, como forma de definir la identidad regional, fue reincorporada cuando Francia no era más una amenaza y los Estados Unidos emergían en el horizonte con una política de intervención en la región, siendo el ejemplo más preocupante el de la participación en la Guerra de Independencia Cubana (1895-1898). El Ariel de José Enrique Rodó, publicado en 1900 es la culminación de esta matriz de pensamiento, el punto máximo del reconocimiento de una latinidad blanca y europea opuesta al utilitarismo anglosajón (RODÓ, 1966). ${ }^{8}$ Sobre esta base se construía la identidad que agrupaba a los del Sur en su relación con los del Norte.

\footnotetext{
${ }^{8}$.- Si bien la imagen de Ariel y de Calibán habían sido utilizadas anteriormente por Paul Groussac, intelectual franco-argentino, y por Rubén Dario, poeta Nicaragüense, Rodó fue quien se apropió de forma más creativa de esta figura. Posteriormente otros importantes intelectuales latinoamericanos retomaron las

Revista Eletrônica da ANPHLAC, ISSN 1679-1061, n.15, p. 155-174, jul./dez. 2013.
} 
Es cierto que esta lectura de una América blanca y europea acosada por el Calibán del Norte no fue aceptada de forma permanente y cosechó opositores en décadas posteriores. La tesis del arielismo latino fue revisada posteriormente por, entre otros, Roberto Fernández Retamar. ${ }^{9}$ La latinidad propuesta por las elites intelectuales de inicios del Siglo XX fue reformulada posteriormente y cambiada de signo, positivando de forma opuesta a aquellos intelectuales, pero manteniendo la herencia de la latinidad. Calibán, el insumiso y rebelde, era la nueva imagen de América Latina que se sublevaba contra la dominación de los Estados Unidos y que no se conformaba con alguna forma de superioridad moral.

Y todavía es posible pensar en el Hispanoamericanismo que representaba la unidad necesaria entre la América Hispana con su antigua metrópolis. Esta matriz de construcción de una identidad sobre la base de la lengua, la religión y la raza excluía a aquellas naciones que no compartían alguna de estas precondiciones. Si esta podía ser una forma de vincular los países de antigua colonización española, recolocando a la vieja metrópoli como un ponto de convergencia, no era una ventaja apreciada por todos estos países. España había dejado de ser metrópoli porque había sido derrotada en Cuba en 1898 y no por voluntad propia. Este era un inconveniente y quien sabe no era el menor de todos para los americanos, principalmente porque la propuesta de España como metrópoli implicaba vincularse a un país decadente y sin el potencial económico de Francia, Inglaterra o Estados Unidos. España, además de ser una potencia decadente, continuaba siendo tan agresiva como en los tiempos de la Colonia, el episodio de la independencia cubana alertaba a todos sobre esta situación. Si los hispanoamericanistas se basaban en valores culturales, España no podía ofrecer nada que la América Ibérica ya no tuviera.

El uso del término "hispanoamericanismo" no deja de ser complejo y problemático. Por un lado tenemos que la primera etapa del panamericanismo, el anterior a las Conferencias Panamericanas es considerado como hispanoamericanismo por algunos autores, debido a que concentraba únicamente a los países de habla hispana. $^{10}$

figuras de Ariel y Calibán según el par de opuestos definidos por Rodó, entre ellos el mexicano José Vasconcelos y el uruguayo José Zorrilla de San Martín, Ver el capítulo 'Los monstruos del latinoamericanismo arielista' de JÁUREGUI, 2008.

9.- El ensayo clásico de Fernández Retamar, 'Calibán', fue revisitado varias veces por él propio, discutiendo diferentes aspectos de esta aproximación. El ensayo original y las posteriores revisitaciones están en FERNÁNDEZ RETAMAR, 2005.

${ }^{10}$.- Para esta lectura del panamericanismo véase BORJA, 2012, P. 1458.

Revista Eletrônica da ANPHLAC, ISSN 1679-1061, n.15, p. 155-174, jul./dez. 2013. 
Si bien la palabra "Hispanoamérica" podía ser un sinónimo de Latinoamérica, como la utilizaba José Vasconcelos (VASCONCELOS, 1931, p. 437), en realidad debía ser entendida como una tentativa de unir a los hispanoparlantes y de reconstituir la Hispanidad para poder expurgar la influencia francesa y poniendo un límite a la preponderancia de los Estados Unidos. ${ }^{11}$ En torno al hispanoamericanismo surgió una polémica que tuvo lugar en mediados de la década de 1920, pero la misma tuvo poca importancia fuera de los círculos intelectuales españoles. Los americanos no consideraron seriamente la propuesta y la revista Martín Fierro, publicada en Buenos Aires (en la que escribían Leopoldo Marechal. Oliverio Girondo y Jorge Luis Borges entre otro), presentó algunas respuestas de forma jocosa y despectiva. ${ }^{12}$

\section{3.- El Panamericanismo en acción}

Lo que nos interesa es que el panamericanismo era una forma de relacionamiento entre las dos Américas y, como fue mencionado anteriormente, no puede ser visto como la consagración del dominio de la América del Norte ni como el ámbito de la resistencia permanente a los Estados Unidos. El panamericanismo imperial que se gestó entre la formulación de la Doctrina Monroe y el Primer Congreso Panamericano lentamente pasó a ser un espacio de convivencia y, principalmente, de negociaciones de todo tipo, sin que desapareciesen completamente las tensiones y los conflictos.

El Primer Congreso Panamericano fue un aprendizaje para todos los países del continente. Los Estados Unidos descubrieron que su poderío económico y militar no podía ser traducido necesariamente en hegemonía política, en cuanto que el resto de los países comprendieron que debían convivir con un vecino poderoso. Las posiciones de los distintos países ante las propuestas del principal miembro del grupo llamaron a la

\footnotetext{
11.- Esta propuesta fue lanzada cuando ya casi no tendría ningún impacto en el debate sobre latinoamericanismo y panamericanismo, pero que merece ser presentada por la tensión que coloca en los otros dos paradigmas. El artículo "Madrid, meridiano intelectual de Hispanoamérica" está disponible en SCHWARTZ, 2002, pp. 594 e ss. Originalmente fue publicado en La Gaceta Literaria Año I, número 8, Madrid, 15 de abril de 1927. El artículo dio paso a un debate sobre el papel de España en la cultura de la América Hispánica, pero no produjo ningún impacto del punto de vista político, ni podría, dada la conturbada situación española. Este hispanismo resurgiría algunos años después en el marco de la Guerra Civil y, más específicamente, como una forma de apoyo a la dictadura instaurada por Francisco Franco.

12.- Para descalificar el debate Jorge Luis Borges y Carlos Mastronardi escribieron en lunfardo con el seudónimo de Ortelli y Gasset: "Espiracusen con plumero y todo, antes que los faje. Che, Meridiano, hacete a un lao, que voy a escupir". Mayores detalles de la polémica en el capítulo destinado a la misma en SCHWARTZ, 2002, pp. 592 e 593. En las páginas siguientes de ese libro son transcriptos algunos de los textos de la misma.
}

Revista Eletrônica da ANPHLAC, ISSN 1679-1061, n.15, p. 155-174, jul./dez. 2013. 
reflexión a todos los involucrados. Los miembros más reticentes del panamericanismo, entre ellos la Argentina, Chile y México, percibieron en el movimiento panamericanista una apertura para sus propias posiciones.

De las tres grandes propuestas que los Estados Unidos realizaron en este encuentro una sola fue acogida: la creación de una oficina de comercio de los Estados Unidos y el resto de América. Mismo así la misma no llegó a ser plenamente implementada. Las otras dos medidas fueron la creación de una Unión Aduanera Común y la creación de un sistema de arbitrajes para los conflictos regionales. En el primer caso fue la Argentina, que mantenía importantes volúmenes de intercambio con Gran Bretaña, la que se opuso por entender que limitaba su política comercial. En el segundo caso fueron los representantes chilenos quienes más fuertemente se opusieron porque entendían que podían perder por la vía diplomática lo que habían obtenido durante la Guerra del Pacífico. El resto de los países percibió que con estas medidas los Estados Unidos impondrían, por un lado, sus intereses económicos y comerciales, y que por otro lado, el puesto de mediador único en los conflictos también recaería en este país (VILLAFAÑE SANTOS, 2008, pp. 315 e 325).

Del punto de vista de las relaciones internacionales el panamericanismo antecede y convive con otras formas de relacionamiento internacional, como las Conferencias Internacionales de Bruselas de 1874 o las de La Haya de 1899 y 1907, y fundamentalmente con el sistema de instituciones y de reuniones de la Sociedad de Naciones que implicaba una convivencia con países fuera del continente y que colocaban otras cuestiones que excedían aquellas que podían ser de importancia central para el continente americano.

Pero la relación con Europa no se manifiesta únicamente con la institucionalidad posterior al Pacto de Versalles ${ }^{13}$, si no que los intereses e influencia de los países europeos se hacían sentir desde el periodo independentista. Por lo tanto, el panamericanismo estaba atravesado por otros intereses que no eran únicamente aquellos de los polos americanos. Sin embargo, vamos a bordar con más detenimiento las cuestiones americanas.

Ya desde la primera Conferencia Panamericana de 1889 en Washington entraron en debate los intereses regionales en relación con los europeos al punto que los

\footnotetext{
${ }^{13}$.- Se denomina de esa forma a la serie de acuerdos firmados entre los gobiernos beligerantes como consecuencia de los resultados de la Primera Guerra Mundial. De estos acuerdos resulta la Sociedad de Naciones y sus organismos afiliados.
}

Revista Eletrônica da ANPHLAC, ISSN 1679-1061, n.15, p. 155-174, jul./dez. 2013. 
representantes británicos en el Río de la Plata se preocupaban por la posibilidad de que los intereses estadunidense los desplazasen de la región. Es por eso que el interés estadunidense de extender su influencia desde el Caribe en dirección al Sur y del Pacífico hacia el Atlántico fue dificultado por la oposición de la Argentina que mantenía fuertes relaciones con la Cancillería Británica. La principal preocupación se centraba en la idea y propuesta estadunidense de crear una zona de comercio común a todas las Américas, lo que era interpretado por los ingleses como un atentado a su posición económica en la región, principalmente en el Cono Sur. Junto con los británicos también reclamaban los alemanes que detenían posiciones económicas en el Cono Sur e intentaban ganar espacio en Centroamérica. ${ }^{14}$

Si para la Argentina el panamericanismo era una amenaza, para otros países era una posibilidad que se abría para el desarrollo nacional o, al menos, una forma de equilibrar las fuerzas económicas existentes. Joaquim Nabuco, el primer embajador brasilero en los Estados Unidos y quien ya había sido representante de ese país en Inglaterra, no veía al panamericanismo como expresión del poderío de los Estados Unidos o como necesariamente negativo, al contrario, debía ser visto como un elemento positivo para las relaciones continentales porque abría la posibilidad de una convivencia continental basada en la presencia de un miembro preponderante que de esa forma inhibía posibles conflictos entre los socios menores. Del punto de vista económico y comercial también el poderío de los Estados Unidos permitiría la proyección de los países consumidores dejando de lado a la antigua metrópoli y creando nuevos y mejores vínculos con la nueva (DENNISON, 2006, pp. 143-152). Desde Brasil, Nabuco pensaba en la complementariedad de los países del continente con el nuevo poder, pero no todos los podían reconocer en esa posición de organización de influencias y de comercio, como ocurría con la Argentina.

Las posiciones divergentes de Brasil y Argentina no hacen más que resaltar la importancia de determinados actores en las relaciones internacionales del período así como las diferencias existentes entre los miembros de la comunidad americana. Estas divergencias en relación al panamericanismo tenían como marco las diferencias existentes entre Brasil y Argentina, que se remontaban a la formación de los Estados Nacionales. Las posiciones en torno al panamericanismo reflejaban esa puja de intereses que se tornó crítica en las primeras décadas del siglo XX, el momento decisivo del

\footnotetext{
${ }^{14}$.- Para conocer algunos de las reclamaciones y preocupaciones de los cónsules británicos y alemanes en el Río de la Plata ver ESCUDÉ e CISNEROS, 2000, Tomo VIII, cap. 40.
}

Revista Eletrônica da ANPHLAC, ISSN 1679-1061, n.15, p. 155-174, jul./dez. 2013. 
panamericanismo. El recurso de la multipolaridad no se limitaba a Brasil y Argentina. Chile, por ej., también tenía sus disputas con Argentina, así como con Perú y Bolivia; lo mismo que Paraguay con Bolivia, Brasil y la Argentina; y podríamos seguir con la enumeración de los conflictos posibles. En el caso del Cono Sur, los actores externos, principalmente Gran Bretaña y Estados Unidos, actuaban como red de contención y apoyo en estas disputas diplomáticas, mas también como incentivadores de conflictos si la ocasión era conveniente (CONDURU, 1998, pp. 64 e 65).

Pero para que el panamericanismo fuese aplicable y aceptable para alguna de las partes involucradas tenía que presentar ventajas concretas, más allá de las simbólicas o posibles en las disputas regionales. Para los países centroamericanos y caribeños las inversiones estadunidenses eran prácticamente las únicas disponibles y el mercado además de ser el más dinámico y activo recibía y estimulaba el consumo de sus productos (CARDOSO, 1992, pp. 185 a 199.). Por su parte, en Uruguay la presencia estadunidense era vista como una forma de contrabalancear la hegemonía británica por medio de las inversiones en un sector clave de la economía como eran los frigoríficos (ODDONE, 1992, p. 129). Lo mismo ocurría con los países de la costa del Pacífico. En las primeras décadas del siglo XX la presencia de los Estados Unidos no fue vista necesariamente como una amenaza, principalmente cuando desplazaba al acreedor habitual en que se había tornado Inglaterra.

Si anteriormente nos referíamos al Ariel de Rodó como una crítica a la modernidad según el padrón estadunidense, basada en la técnica y en el utilitarismo, no es menos cierto que los avances tecnológicos estimulaban el vínculo con este país porque oferta una serie de avances científicos y tecnológicos, productos industriales y mercado para buena para de los productos de los demás países americanos. Es por eso que durante el periodo más acentuado del panamericanismo, entre finales del Siglo XIX y las primeras décadas del Siglo XX, los Estados Unidos desarrollarían una serie de iniciativas con el objetivo de construir una visión positivada sobre su modo de vida que era exportado al resto del continente. Si bien estas iniciativas tenían objetivos imperiales, en el sentido de lograr una hegemonía y un predominio informal, las mismas implicaban la constitución de instituciones, junto con una batería de acciones culturales

Revista Eletrônica da ANPHLAC, ISSN 1679-1061, n.15, p. 155-174, jul./dez. 2013. 
y materiales que permitiesen a los demás países del continente reconocerse en esa relación como necesariamente vinculados y reconocidos por el Norte. ${ }^{15}$

Una serie de iniciativas integradoras fueron puestas en práctica, si bien las mismas se vinculaban con los intereses de los Estados Unidos, también eran importantes para los demás países del continente. Entre estas iniciativas están la construcción de un ferrocarril transcontinental, la autopista panamericana, una línea de aviación transcontinental y el Canal de Panamá. Si bien no todas se completaron o se completaron parcialmente, estos proyectos representaban la materialización del panamericanismo, la consecución práctica de los debates que se sucedían en las Conferencias Panamericanas. ${ }^{16}$

Además de la tecnología y capitales aplicados a la integración regional no podemos dejar de mencionar el esfuerzo político representado por las múltiples Conferencias Panamericanas que tuvieron lugar desde 1889 hasta 1948. En estas conferencias los países americanos colocaron sus perspectivas, discutieron sus afinidades y sus doctrinas de como relacionarse, se pusieron de acuerdo en algunas cuestiones o confrontaron, según el momento. Lo que nos llevaba a reafirmar la importancia dada a estos elementos es que los países miembros disputaban el hecho de ser la sede de la conferencia y participaban de la misma discutiendo las propuestas y pensando en un sistema americano de legislación y de comercio. Es por eso que no podemos dejar de lado que este era un fórum para escuchar y ser escuchado, para peticionar y confrontar buscando la aprobación y la mayor cantidad de apoyos posibles. Por lo tanto, reafirmamos de que en nuestra lectura del panamericanismo el mismo no puede ser visto como la simple expresión de la hegemonía estadunidense o de sus proyectos imperialistas y si como un espacio aceptado y consensuado por todos los miembros.

\section{4.- El Universalismo de la Sociedad de Naciones y las Américas}

Si el Panamericanismo partía de la Realpolitik ${ }^{17}$ y de las prácticas y capacidad de movilizar apoyos para crear consensos antes que normas. Esta era una gran diferencia

\footnotetext{
${ }^{15}$.- Ricardo Salvatore nos muestra la opción del convencimiento y el dialogo con América del Sur (SALVATORE, 2004, p. 26).

${ }^{16}$.- Para mayores detalles de estos emprendimientos estadunidenses y el sentido de los mismos en el contexto panamericano SALVATORE, 2006, pp. 662-691.

${ }^{17}$.- El término Realpolitik es utilizado principalmente en el ámbito de las relaciones internacionales con el intuito de alcanzar intereses concretos por parte de cada país donde el interés nacional son superiores a los principios éticos o morales. Para mayores datos sobre la Realpolitik ver BORJA, 2012, p. 1662-1663. Si

Revista Eletrônica da ANPHLAC, ISSN 1679-1061, n.15, p. 155-174, jul./dez. 2013.
} 
con el universalismo propuesto, por ej., por la Organización Internacional del Trabajo o por la Sociedad de Naciones. El universalismo partía de una serie de principios y de normas calcadas en la legislación y en los intereses de los principales países europeos, y esto que podía funcionar en el espacio europeo y en sus colonias debido a las posibilidades de ejercer presión o coerción sobre los países más próximos o afines a la civilización encarnada en estas instituciones. Pero para los países americanos estos principios podían resultar poco recomendables y aplicables en contados lugares de sus propios territorios, principalmente en las capitales o en las regiones poblacionalmente europeizadas. Para el resto del territorio americano era preciso considerar las formas de relacionamiento y las legislaciones preexistentes, principalmente en territorio mayoritariamente ocupado por población indígena o en las zonas rurales ocupadas por poblaciones tradicionales.

El ideal de una justicia, de una legislación o de principios de convivencia universales, comunes a todos los pueblos en cualquier lugar o en cualquier tiempo histórico derivaban de la justicia, la legislación y de los principios europeos. Lo que para Europa era un acuerdo que representaba finalmente la posibilidad de alcanzar la estabilidad y la paz dejada atrás durante la Gran Guerra, para América parecía ser una intromisión, una obligación que no era vista como necesaria. Sus propias cuestiones muchas veces se chocaban con los intereses representados en Versalles, primero, y en Ginebra, después. ${ }^{18}$

Un ejemplo de este conflicto entre universalismo y regionalismo es el caso de la relación entre Argentina y la Sociedad de Naciones. Argentina fue convidada a participar de la Sociedad y su aceptación estaba condicionada a que los neutrales y derrotados pudieran participar. La posición argentina se sustentaba en el principio diplomático creado en 1869 para superar la crisis regional abierta por la Guerra del Paraguay, denominado de Doctrina Varela, por el canciller argentino Mariano Varela, y

bien el panamericanismo es una forma de relacionarse que prioriza los consensos y los debates antes que los conflictos no podemos dejar de lado que todos los países priorizaban sus propios intereses antes que principios doctrinarios. De esa forma se explica que determinados países oscilasen entre los distintos polos existentes en cada una de las Conferencias.

${ }^{18}$.- Este universalismo ha sido cuestionado por los teóricos de los estudios poscoloniales. Sin entrar en detalles, porque no es el objetivo de este artículo, no podemos dejar de mencionar el hecho de que el universalismo es la tentativa de inscribir identidades y valores propios del Occidente Europeo en otras realidades espacio-culturales. Europa se impuso como universal al considerarse como el sujeto teórico de todas las Historias. De esta forma, el universalismo propuesto por la OIT, y por otras instancias ginebrinas, deben ser considerados como una forma de eurocentrismo basado en la imposición de la experiencia previa de los países europeos ante la reciente inclusión de los países americanos al sistema de producción capitalista y, más específicamente, al capitalismo industrial. Para una crítica al universalismo europeo ver BABBHA, 2010, pp. 270 e 271; e PRAKASH, 1994, p. 1484.

Revista Eletrônica da ANPHLAC, ISSN 1679-1061, n.15, p. 155-174, jul./dez. 2013. 
que puede ser resumido como: "La victoria no da derechos". O sea que la magnanimidad con un pueblo derrotado permitiría reconstruir las relaciones más rápidamente y superar los conflictos si se contaba con el acuerdo de los derrotados. ${ }^{19}$ Esta Doctrina contrariaba los acuerdos de Paz de Versalles que despedazaron territorialmente a las potencias centrales, sin contar las pesadas compensaciones económicas que condicionaron la recuperación de estos espacios. La Argentina ingresó a la Sociedad en el momento de su creación y la abandonó en octubre de 1920 en desacuerdo con la continuidad de la exclusión de neutrales y derrotados. Dos principios generales entraron en conflicto: el de la Argentina y el de las naciones europeas victoriosas, y sobre este segundo principio fue que se construyó la Sociedad de Naciones.

La universalidad de la Sociedad fue cuestionada desde el mismo momento de su creación por varios países americanos. Argentina, después del entredicho de la neutralidad ya mencionado, permaneció alejada hasta 1933. Brasil abandonó la Sociedad en 1926 por no haber sido contemplado su pedido de formar parte del Consejo de Seguridad. México solo fue aceptado en 1931 y los Estados Unidos, cuyo presidente Woodrow Wilson había sido uno de sus más fervientes entusiastas, nunca ratificaron el tratado de fundación de la Sociedad. Para América, entonces, esta organización no parecía ser tan importante y sus preocupaciones se encausaban mucho más por la vía de las relaciones regionales. Los vínculos con los países Europeos se resolvían a través de las relaciones bilaterales.

La universalidad, en este caso, dependía mucho más de una lectura de la realidad ante situaciones particulares y no en un principio aceptado por todos. Por lo tanto, la universalidad dependía mucho más de intereses concretos, que eran los intereses europeos, y esto se extendería a otros campos, como por ej. el derecho del trabajo donde el universalismo europeocéntrico chocaba de frente con las prácticas regionales, las necesidades de industrias en crecimiento o, en algunos casos, con su inexistencia, la importancia del trabajo agrícola, del trabajo familiar y comunitario o con las formas de uso del trabajo indígena por la vía del uso directo del mismo o por medio de los tributos exigidos en trabajo. Es preciso nuevamente resaltar que aún con la elección de mantener las relaciones bilaterales como prioridad para los diversos países del continente

${ }^{19}$.- Ver: ESCUDÉ, C. e CISNEROS, A. Op. Cit., Tomo VI, cap. 31.

Revista Eletrônica da ANPHLAC, ISSN 1679-1061, n.15, p. 155-174, jul./dez. 2013. 
americano. Tanto el panamericanismo, el latino-americanismo o el universalismo deben ser vistos como opciones tácticas antes que como decisiones estratégicas.

Únicamente los Estados Unidos pueden ser considerados como detentores de una estrategia y como interesados de mantener el sistema panamericano en actividad. Como mencionamos, este país no se afilió a la Sociedad y fue tardío su ingreso en otros organismos, como es el caso de la OIT a la que ingresó en 1936, el panamericanismo era la vía de integración excluyente y en ella cifraban sus expectativas para mantener su comercio activo durante las crisis económicas o como salvaguarda en momentos de crisis militar. En América Latina estaban sus aliados más fieles y sus enemigos menos peligrosos. A medida que avanzaba el siglo XX las ilusiones hegemónicas de Argentina no podían ser vistas como una amenaza seria para los Estados Unidos dentro del marco de negociaciones y conversaciones permanentes de la institucionalidad panamericana. Argentina, Chile, Brasil o México oscilaban con otros polos mucho más como una forma de tener una mejor posición relativa a la hora de negociar con los Estados Unidos que como una forma de confrontar decisivamente con estos.

El panamericanismo, entonces, no puede simplemente ser visto como un ideal de difícil consecución. Por el contrario, el panamericanismo fue puesto en marcha, de forma imperfecta, negociada y con grandes dificultades producto de la desconfianza entre los países americanos. Y así y todo fue un marco importante para las relaciones entre las naciones del continente Americano.

\section{BIBLIOGRAFIA}

BABBHA, Homi. O local da Cultura. Belo Horizonte: Ed. UFMG, 2010.

BERGER, Mark T. Civilising the South: The US Rise to Hegemony in the Americas and the Roots of 'Latin American Studies' 1898-1945. IN: Bulletin of Latin American Research, London, Society for Latin American Studies, Vol. 12, No. 1, Jan. 1993.

BETHELL, Leslie. Brazil and Latin America. IN: Journal of Latin American Studies. Cambridge, Cambridge University Press, N 42 / 3, 2010.

BORJA, Rodrigo. Panamericanismo. IN: Enciclopedia de la política, T. II. México, FCE, 2012.

CARDOSO, Ciro Flamarion. América Central: la era Liberal. C. 1870-1930. IN: BETHELL, L. (ed.). Historia de América Latina. Vol 9. Barcelona: Crítica, 1992.

Revista Eletrônica da ANPHLAC, ISSN 1679-1061, n.15, p. 155-174, jul./dez. 2013. 
CONDURU, Guilherme Frazão. O subsistema Americano, Rio Branco e o ABC. IN: Revista Brasileira de Política Internacional. Brasília, Instituto Brasileiro de Relações Internacionais, 41/2, 1998.

DENNISON, Stephanie. Joaquim Nabuco. Monarchism, Panamericanism and NationBuilding in the Brazilian Belle Epoque. Oxford, Peter Lang, 2006.

ESCUDÉ, Carlos e CISNEROS, André. Historia de las Relaciones Exteriores de Argentina. Buenos Aires: GEL editores, 2000.

FERNÁNDEZ RETAMAR, Roberto. Todo Calibán. Buenos Aires: Clacso, 2005.

GRANDIN, Greg. Your Americanism and Mine: Americanism and Anti-Americanism in the Americas. IN: The American Historical Review, Washington, American Historical Association, Vol. 111, No. 4, October 2006.

JÁUREGUI, Carlos. Canibalia. Canibalismo, Calibanismo, antropofagia cultural y consumo en América Latina. Madrid: Iberoamericana, 2008.

MACDONALD, C. A. The Politics of Intervention: The United States and Argentina, 1941-1946. IN: Journal of Latin American Studies, Cambridge, Cambridge University Press, Vol. 12, No. 2, Noviembre de 1980.

MACMILLAN, Margaret. Paris 1919: Six months that changed the world. New York: Random House, 2002.

MORGENFELD, Leandro. Vecinos en conflicto. Argentina y Estados Unidos en las Conferencias Panamericanas (1880-1955). Buenos Aires: Peña Lillo, 2011.

ODDONE, Juan A. La formación del Uruguay moderno. C. 1870-1930. IN: BETHELL, L. (ed.). Historia de América Latina. Vol 10. Barcelona: Crítica, 1992.

OLIVA CAMPOS, Carlos. Estados Unidos-América Latina y el Caribe: entre el panamericanismo hegemónico y la integración independiente. IN: CRISORIO, Carolina et al. Historia y Perspectiva de la Integración Latinoamericana. Morelia: AUNA Universidad Michoacana San Nicolás de Hidalgo, 2000.

PRAKASH, Gyan. Subaltern Studies as Postcolonial Criticism. IN: The American Historical Review. Chicago, The Chicago University Press - American History Association, Vol. 99, No. 5, Dec., 1994.

RODÓ, José Enrique. Ariel. Buenos Aires: Kapelusz, 1966.

SALVATORE, Ricardo. Imágenes de un Imperio. Estados Unidos y las formas de representación de América Latina. Buenos Aires: Sudamericana, 2004.

Revista Eletrônica da ANPHLAC, ISSN 1679-1061, n.15, p. 155-174, jul./dez. 2013. http://revista.anphlac.org.br/ 
SALVATORE, Ricardo. Imperial Mechanics: South America's Hemispheric Integration in the Machine Age. IN: American Quarterly 58/3. Baltimore, The Johns Hopkins University Press, September 2006.

SCHWARTZ, Jorge. Las vanguardias latinoamericanas. Textos programáticos y críticos. México, FCE, 2002.

SEXTON, Jay. The Monroe Doctrine. Empire and Nation in Nineteenth-Century America. New York: Hill and Gang, 2011.

TOCQUEVILLE, Alexis. La democracia en América. Buenos Aires: Hyspamérica, 1985.

VASCONCELOS, José. Panamericanism or Hispanoamericanism. Books Abroad. Oklahoma, Board of regents of Oklahoma University, Vol. 5, No. 4, October 1931.

VILLAFAÑE SANTOS, Luis Claudio. Las relaciones interamericanas. In: AYALA FRANCO, Enrique y POSADAS CARBÓ, Eduardo. Historia General de América Latina. Vol. VII. París: Unesco / Trotta, 2008.

Revista Eletrônica da ANPHLAC, ISSN 1679-1061, n.15, p. 155-174, jul./dez. 2013. http://revista.anphlac.org.br/ 